\title{
Ubicación óptima de reconectadores y fusibles en sistemas de distribución
}

\section{Optimal location of reclosers and fuses in distribution systems}

\author{
Juan Pablo Gallego-Londoño \\ Ing. Electricista \\ Universidad Tecnológica de Pereira \\ Pereira, Colombia \\ jupagallego@utp.edu.co \\ Ricardo Alberto Hincapié-Isaza \\ M.Sc. Ing. Eléctrica \\ Universidad Tecnológica de Pereira \\ Pereira, Colombia \\ ricardohincapie@utp.edu.co
}

\author{
Oscar Danilo Montoya-Giraldo \\ M.Sc. Ing. Eléctrica \\ Universidad Tecnológica de Pereira \\ Pereira, Colombia \\ odmontoya@utp.edu.co \\ Mauricio Granada-Echeverri \\ Ph.D. en Ingeniería \\ Universidad Tecnológica de Pereira \\ Pereira, Colombia \\ magra@utp.edu.co
}

\begin{abstract}
Resumen- En este artículo se presenta una metodología para resolver el problema de localización óptima de reconectadores y fusibles en sistemas de distribución de energía, para mejorar indicadores de confiabilidad asociados a la frecuencia con la que inciden las fallas transitorias y permanentes en los sistemas de distribución. Para formular el problema se plantean dos modelos matemáticos no lineales de tipo binario que consideran la ubicación de reconectadores y fusibles bajo dos escenarios de coordinación, denominados fuse save scheme y fuse blow scheme. El primer modelo minimiza el índice SAIFI y el segundo minimiza el índice MAIFI. El conjunto de restricciones es el mismo para ambos modelos, el cual considera características técnicas y operativas. Como estrategia de solución se propone una metodología que considera dos esquemas diferentes de solución: la primera emplea el optimizador comercial GAMS y la segunda usa como técnica de solución un algoritmo genético de Chu \& Beasley (AGCB). Con el fin de verificar la validez de ambas estrategias, estas son aplicadas a dos sistemas de prueba de la literatura especializada, comparando los resultados obtenidos.
\end{abstract}

Palabras clave—Algoritmo genético, fusibles, confiabilidad, modelo matemático no lineal, sistemas de distribución, reconectadores.

Abstract- In this article a methodology to resolve the problem of optimal location of reclosers and fuses in power electric distribution systems is presented, to improve reliability indices associated to the periodicity of the transient and permanent failures in the distribution systems. To formulate the problem, two non-linear binary mathematical models are posed that consider the location of reclosers and fuses under two scenarios of coordination, denominated "fuse save scheme" and "fuse blow scheme". The first model minimizes SAIFI in- dex, and the second model minimizes MAIFI index. The set of constraints is the same for both models, which considers technical and operative characteristics. As solution strategy a methodology is proposed, which considers two different schemes of solution: the first scheme uses the commercial optimizer GAMS and the second scheme uses as solution technique a Chu \& Beasley genetic algorithm (CBGA). In order to verify the validity of both strategies, these are executed in two tests systems from the specialized literature, comparing the results obtained.

Keywords- Genetic algorithm, fuses, reliability, nonlinear mathematical model, distribution systems, reclosers.

\section{INTRODUCCIÓN}

Los sistemas de distribución son los encargados de entregar energía a los usuarios de un sistema eléctrico, garantizando criterios de calidad, confiabilidad y continuidad al menor costo posible. Sin embargo, la operación de estos sistemas está sujeta a perturbaciones como fluctuaciones de voltaje, contenido armónico de las ondas de voltaje y corriente, variaciones de frecuencia, fallas en los elementos de la red y cortocircuitos en el sistema, lo que ocasiona algunas veces indisponibilidad en el servicio. Debido a esto los entes reguladores exigen cada vez más a los operadores de red, con el fin de mejorar las características operativas de los sistemas de distribución y garantizar un mejor servicio para los usuarios. 
Como respuesta a las exigencias de los entes reguladores y a las condiciones operativas de los sistemas de distribución, los operadores de red han desarrollado diferentes estrategias que permitan dar cumplimiento a estas exigencias. De acuerdo con esto, en los últimos años se ha empleado la ubicación de elementos de protección como reconectadores y fusibles sobre las redes de distribución [1], debido a su efectividad para proteger estos sistemas, su bajo costo comparado con equipos de protección en subestaciones y su flexibilidad para coordinarlos.

Tradicionalmente los reconectadores se han empleado como protección primaria a la salida de la subestación o como protección de secciones de la red al estar ubicada a lo largo de su recorrido. Debido a la característica de reconexión que presenta este elemento, los índices de confiabilidad del sistema mejoran significativamente debido a la propiedad de discriminar fallas permanentes de temporales, a partir de la duración que estas presentan. Por otro lado, los fusibles se han empleado tradicionalmente como protección a lo largo de las redes de distribución, teniendo como característica principal su operación ante cualquier tipo de falla (permanente o temporal). Esta situación puede empeorar en algunos casos los indicadores de confiabilidad, ya que la actuación del fusible puede generar indisponibilidad del servicio por una operación permanente por fallas temporales.

De acuerdo con esto, los reconectadores pueden ser empleados en conjunto con los fusibles, con el fin de garantizar que estos últimos no actúen ante la presencia de fallas temporales. Esta característica operativa se logra empleando un esquema conocido como salvamento de fusibles, en el cual se garantiza que siempre operen primero los reconectadores y después los fusibles.

A pesar de que la operación conjunta de reconectadores y fusibles mejora indicadores de confiabilidad del sistema, encontrar el punto de ubicación de estos elementos sobre las redes genera un problema adicional, debido a la cantidad de ramificaciones y tramos de red que contiene un sistema de distribución, a los diferentes tipos de carga y a la longitud que posee, lo que ocasiona un problema de tipo combinatorial debido a la gran cantidad de alternativas de solución posibles. Adicionalmente, una inadecuada localización de estos elementos puede presentar los siguientes inconvenientes: indisponibilidad en el servicio, sobrecostos a causa de exceso de ubicación de elementos, fluctuaciones de voltaje, aumento del nivel de energía no servida y penalizaciones debido al incumplimiento de indicadores de confiabilidad.

De acuerdo con lo anterior, la ubicación óptima de elementos de protección es un tema que ha cobrado gran interés en el sector eléctrico, en especial para los operadores de red, haciendo de esta temática un área de investigación en desarrollo, que aún falta por explorar y que además presenta una gran cantidad de investigaciones y proyectos orientados a mejorar o solucionar este problema. En general, el impacto que tiene en los usuarios la ubicación óptima de los diferentes dispositivos de protección está relacionado con el mejoramiento de los índices que corresponden a la frecuencia y la duración de las interrupciones [2]-[3]. A continuación se presentan los trabajos más relevantes en torno a esta temática.

En 1997 Kagan et al. [4] presentaron una metodología para minimizar la cantidad de energía no servida en redes de distribución primaria, a través de la sustitución e inserción de dispositivos de protección. La metodología propuesta considera algunos aspectos como la reconfiguración de la red, representación de la red por zonas de carga y análisis del costo/beneficio de la instalación de estos dispositivos.

Soudi et al., en 1998 [5], describieron el problema con un modelo matemático binario, el cual permite identificar el tipo y la ubicación de los dispositivos de protección en redes de distribución. El algoritmo propuesto usa técnicas heurísticas para minimizar el tiempo de cómputo. La función objetivo empleada busca minimizar el índice de frecuencia promedio de interrupciones permanentes del sistema (SAIFI). Estos mismos autores en 2001 [6] plantean el problema como un modelo no lineal binario con restricciones lineales. La formulación de este trabajo supone la división de la red de distribución en cuatro categorías diferentes: un alimentador principal y tres tipos de laterales. En la aplicación de la metodología solo el alimentador principal y ciertos tramos de red son explícitos para ser optimizados. Además los autores proponen lograr un razonable equilibrio entre la disminución del SAIFI y un aumento en el índice de frecuencia promedio de interrupciones 
momentáneas del sistema (MAIFI) para determinar dónde es posible usar un esquema de salvamento de fusibles. En ese mismo año Kagan et al. [7] presentaron un modelo para determinar y minimizar los índices de duración y frecuencia de las fallas para una red de distribución primaria, en la que los índices de calidad del servicio son considerados a través de las tasas medias de falla y el tiempo de restauración del servicio.

Silva en 2002 [8] plantea una formulación matemática para el problema de ubicación de dispositivos de protección, que considera la posibilidad de adicionarlos en lugares estratégicos, buscando mejorar el índice de confiabilidad de la red. El modelo matemático propuesto corresponde a un problema de programación no lineal con variables de tipo binario, el cual considera una función objetivo no lineal con un conjunto de restricciones lineales. En la solución del problema se emplea un algoritmo evolutivo. Este mismo autor en 2005 [9] propone un modelo de programación no lineal entero mixto, con el fin de resolver la ubicación de seccionalizadores y dispositivos de protección en redes de distribución. Esta propuesta tiene como objetivo reducir el tiempo de desconexión de la red, debido a fallas temporales y minimizar el número de consumidores afectados, garantizando confiabilidad y bajos costos, logrando de esta manera un aumento en las utilidades de las empresas y el cumplimiento riguroso de la reglamentación establecida por parte de los organismos de control.

Moreti en 2007 [10] presenta una metodología para ubicar dispositivos de protección en sistemas de distribución primaria, considerando generación distribuida. El modelo matemático supone la minimización de la energía no servida sujeto a un conjunto de restricciones técnicas y operativas, empleando una técnica heurística para su solución.

Reyes et al. [11] presentan una metodología para la ubicación óptima de reconectadores automáticos en sistemas de distribución con el objetivo de minimizar la frecuencia media de interrupción del servicio bajo la reglamentación vigente en Chile. Son consideradas en el análisis redes radiales con carga uniformemente distribuida y se realiza una aproximación lineal en el modelado matemático del problema.
Kim et al. en 2010 [12] proponen el análisis para la ubicación y coordinación óptima de fusibles y reconectadores en sistemas de distribución empleando una técnica heurística. Para verificar el comportamiento de las protecciones después de haber sido ajustadas, los autores emplean sistemas de prueba reales usando el software PSCAD/EMTDC. En este mismo año Ferreira et al. [13] emplean optimización multi-objetivo para minimizar índices como TIEPI (Tiempo de Interrupción Equivalente de la Potencia Instalada), SAIDI y MAIFI a través de la asignación óptima de elementos de protección. Para obtener una solución se emplea el algoritmo genético de ordenamiento no dominado NSGA II (Nondominated Sorting Genetic Algorithm II).

Darabi et al. en 2011 [14] plantean un método de programación binaria para la ubicación de dispositivos de protección como reconectadores, fusibles y seccionalizadores, empleando el índice SAIDI. EI método propuesto es evaluado a partir de la implementación en el software Visual Studio. Este mismo año Toro et al. [15] proponen un modelo matemático de tipo binario para la ubicación de elementos de protección a partir de una técnica que consiste en dividir el circuito de distribución en una sección principal y ramales laterales. En este trabajo se busca mejorar los índices de confiabilidad SAIFI y ASIFI. EI modelo es resuelto utilizando el paquete de optimización GAMS.

Ferreira et al. en 2012 [16] mostraron un nuevo modelo del problema con una representación no lineal de tipo binario, cuyo objetivo es minimizar los índices SAIFI y ASIFI en un alimentador de distribución radial. El modelo mostrado no requiere decisiones heurísticas sobre la división del alimentador y clasificación de las ramas. Para resolver el modelo propuesto se usa una técnica de optimización basada en el algoritmo de Branch-and-Bound. En este mismo año, Peñuela en su tesis doctoral [17] propone un modelo matemático para determinar el impacto de fuentes de generación distribuida en sistemas de distribución de energía, sobre el planeamiento de sistemas de distribución, considerando cuatro tipos de dispositivos de protección (reconectadores, seccionalizadores, fusibles y relés). El modelo es resuelto mediante la técnica de optimización multi-objetivo NSGA II y se valida en diferentes 
sistemas de prueba de la literatura especializada. También en el año 2012 Tio et al. [18] presentan un modelo de programación no lineal binaria para los indicadores SAIFI y MAIFI en función de la ubicación de reconectadores y fusibles en un sistema de distribución de energía con características radiales. En el modelo es tenido en cuenta el comportamiento del sistema con y sin salvamento de fusibles. El modelo es resuelto a través de un paquete de optimización comercial y es construido un frente óptimo de Pareto con una metodología de ponderación en las funciones objetivo.

Abdi et al. en 2013 [19] plantean una metodología basada en el análisis costo/beneficio para la ubicación óptima de reconectadores y seccionalizadores, cuya función objetivo considera la minimización de los costos de la confiabilidad de los sistemas de distribución. Además presentan un método híbrido basado en enjambre de partículas y simulación de Monte Carlo para solucionar el problema.

Con el fin de solucionar el problema de la ubicación óptima de reconectadores y fusibles en sistemas de distribución para el mejoramiento de índices de confiabilidad, en este trabajo se propone una metodología que considera dos estrategias diferentes de solución: la primera emplea un optimizador comercial y la segunda usa como técnica de solución un AGCB; se decidió emplear esta metaheurística debido a sus resultados satisfactorios en trabajos similares. Para describir el problema se emplean dos modelos matemáticos no lineales de tipo binario. El primero minimiza el índice SAIFI y el segundo el índice MAIFI. El conjunto de restricciones es el mismo para ambos modelos, el cual considera características técnicas y operativas. Con el fin de verificar la validez de ambas estrategias, estas son aplicadas a dos

\section{1 Nomenclatura}

Conjuntos, índices y parámetros sistemas de prueba de la literatura especializada, comparando los resultados obtenidos.

Este trabajo es organizado de la siguiente forma. En la sección 2 se describe la formulación y descripción del problema, además de los modelos matemáticos con sus funciones objetivo y sus respectivas restricciones. En la sección 3 se describe el AGCB y sus principales características. En la sección 4 se presenta la metodología propuesta para solucionar el problema. La sección 5 muestra la aplicación de la metodología propuesta en dos sistemas de distribución de características reales. Finalmente se encuentran las conclusiones y recomendaciones derivadas de este proyecto, además de la bibliografía empleada.

\section{MODELADO MATEMÁTICO}

La ubicación de los dispositivos de protección en sistemas de distribución corresponde a un problema de alta complejidad matemática, ya que, para minimizar los indicadores de confiabilidad a partir de la localización de los elementos de protección sobre la red, se requiere de un modelo matemático que evalúe dichos indicadores en función del número de usuarios, las tasas promedio de fallas en los tramos de red y la disposición del esquema de protecciones en el sistema de distribución. Por las condiciones anteriores, el modelo matemático resultante es de tipo no lineal binario y de característica polinómica, en el cual es considerada la ubicación de fusibles y reconectadores, bajo dos esquemas de operaciones en forma simultánea. En la sección 2.1 se presenta la nomenclatura empleada en la formulación de las funciones objetivo y cada uno de los términos que las componen, además de las restricciones generales del problema.

\footnotetext{
$F(i): \begin{aligned} & \text { Función que retorna el conjunto de tramos de red en el camino de la subestación al tramo de red } \\ & i \text {, incluyendo el tramo inmediatamente después de la subestación y excluyendo el tramo de red } i \text {. }\end{aligned}$

$G(j, i)$ : Función que retorna el conjunto de tramos de red en el camino de la línea $j$ a la línea $i$, excluyendo la línea $j$ e incluyendo la línea $i$.

$G^{\prime}(j, i)$ : Función que retorna el conjunto de tramos de red en el camino de la línea $j$ a la línea $i$, excluyendo tanto la línea $j$ como la línea $i$.

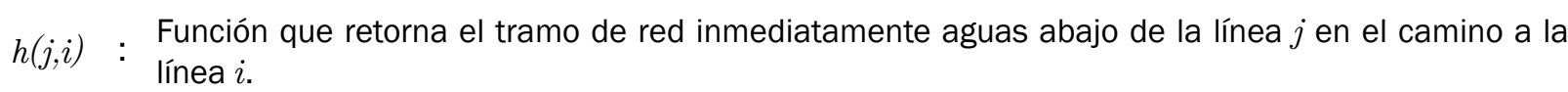




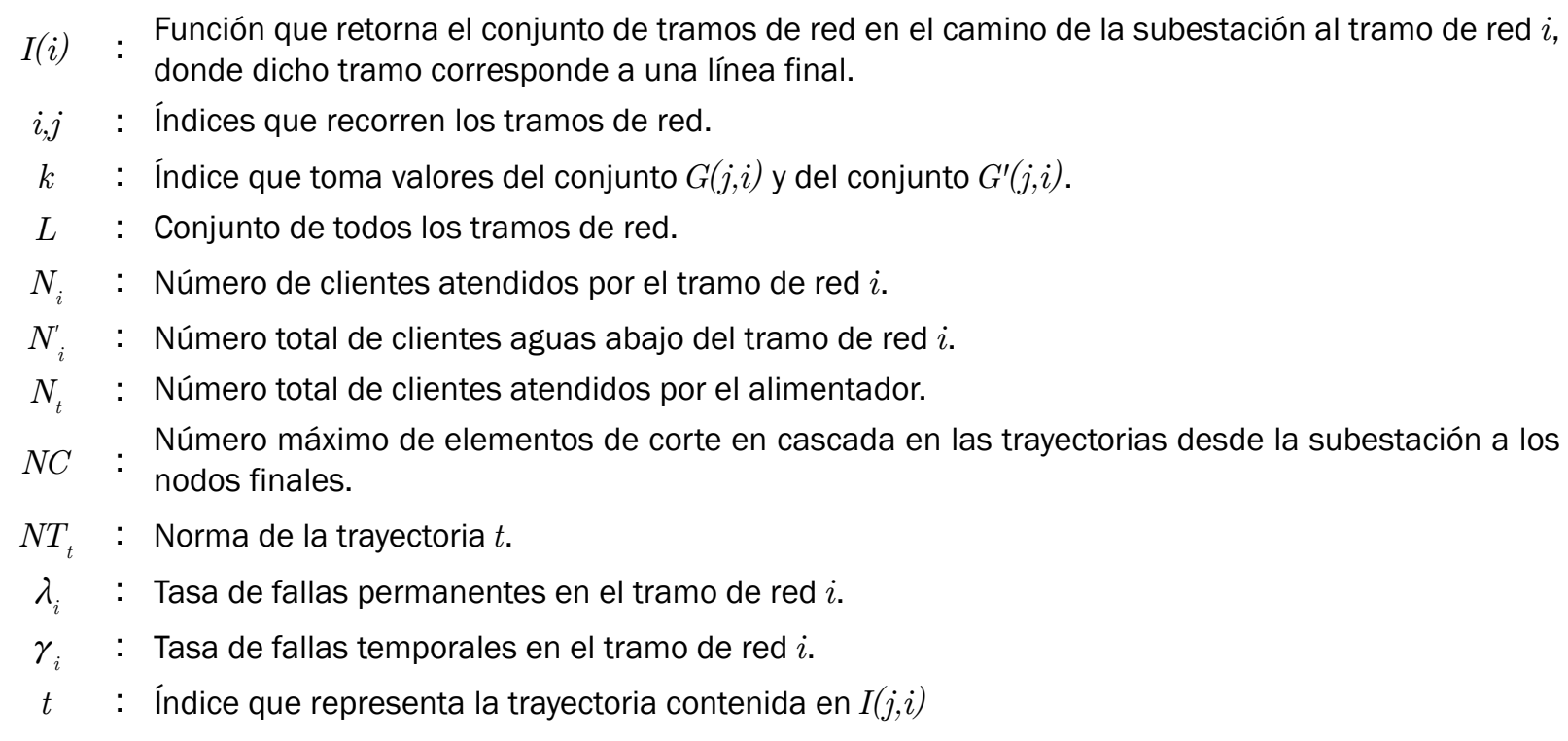

Variables binarias

$x_{i 1} \quad$ : Define la instalación $\left(\mathrm{x}_{i 1}=0\right)$ o no $\left(\mathrm{x}_{i 1}=1\right)$ de un reconectador en el tramo de red $i$.

$x_{i 2} \quad$ : Define la instalación $\left(\mathrm{x}_{i 2}=0\right)$ o no $\left(\mathrm{x}_{i 2}=1\right)$ de un fusible en el tramo de red $i$.

$x_{i 4}$ : Define la instalación $\left(\mathrm{x}_{i 4}=0\right)$ o no $\left(\mathrm{x}_{i 4}=1\right)$ de un fusible con esquema de salvamento en el tramo de red $i$.

Con el fin de interpretar de manera adecuada la nomenclatura presentada, considérese el sistema un sistema de distribución radial como el presentado en la sección de aplicación y resultados (ver Fig. 4 y Tabla I). Para este sistema se analizan, a manera de ejemplo, los tramos de red 1, 2 y 4 . El tramo de red tiene 5400 usuarios por lo que $N_{1}^{\prime}=5400$, así mismo $N_{2}^{\prime}=3400$ y $N_{4}^{\prime}=800$. Para la función $F(i)$ en tramo de red 1 , según la definición dada se genera un conjunto vacío $F(1)=\{\}$, mientras que para el tramo de red 2 el conjunto ya no es nulo $F(2)=\{1\}$ y $F(4)=\{1,2,3\}$. Al analizar la función $G(j, i)$ se tiene que $G(1,2)=\{2\}, G(1,4)=$ $\{2,3,4\}$ y $G(2,4)=\{3,4\}$. Por otro lado, si se observa la función $G^{\prime}(j, i)$, en su definición se indica que en el camino de la línea $j$ hacia la línea $i$ se excluyen ambos elementos por lo cual se llega a $G^{\prime}(1,2)=$ \{\}$, G^{\prime}(1,4)=\{2,3\}$ y $G^{\prime}(2,4)=\{3\}$. Considerando ahora la función $h(j, i)$, esta retorna el tramo inmediatamente aguas debajo de $j$ en su camino hacia $i$, por lo cual se tiene $h(1,2)=\{2\}, h(1,4)=$ $\{2\}$ y $h(2,4)=\{3\}$. Nótese que $G(4,2)=\{\}, G^{\prime}(4,2)=$ \{\} y $h(4,2)=\{\}$ son vacíos puesto que el tramo de red $j$ siempre debe estar aguas arriba de $i$ y en este caso no se cumple está condición. Para el caso de la función $I(i)$, esta sólo tiene sentido para los tramos de red finales, es decir, para el caso de ejemplo, los tramos $6,7,8$, y 9 . Siendo así, $I(6)=\{1,5,6\}, I(7)=\{1,2,7\}, I(8)=\{1,2,3,8\}$ e . 9) $=\{1,2,3,4,9\}$.

\subsection{Función objetivo SAIFI}

El índice SAIFI representa el promedio de las interrupciones por cliente y se calcula como el cociente entre la suma total de interrupciones percibidas por cada cliente sobre el número total de clientes atendidos. Este indicador es de gran utilidad para las empresas distribuidoras de energía eléctrica porque representa la calidad media del sistema. En forma general, el índice SAIFI (1) puede calcularse según [18], como se muestra a continuación:

$$
S A I F I=\frac{\sum_{i \in L} \lambda_{i} * N_{i}}{N_{t}}=\frac{A_{q}}{N_{t}}
$$

El término $A q$ en la parte derecha de (1) se puede expresar como una ecuación no lineal binaria 
en términos de la ubicación de los dispositivos de protección, como se presenta en (2).

$$
S A I F I=\frac{1}{N_{t}}\left(\begin{array}{c}
\sum_{i \in L} \lambda_{i} * N_{i}^{\prime}+\sum_{i \in L} \gamma_{i}\left(1-x_{i 2}\right) * N_{i}^{\prime}+ \\
\sum_{i \in L} \lambda_{i} \sum_{j \in F(i)}\left(N_{j}^{\prime}-N_{h(j, i)}^{\prime}\right) \prod_{k \in G(G, i)} x_{k 1} x_{k 2} x_{k 4}+ \\
\sum_{i \in L} \gamma_{i} \sum_{j \in F(i)}\left(1-x_{j 2}\right) * N_{j}^{\prime} \prod_{k \in G(j, i)} x_{k 1} x_{k 2}
\end{array}\right)
$$

De la ecuación (2) se tiene que el primer y el tercer término corresponden a la influencia de las fallas permanentes en el SAIFI y el segundo y cuarto término corresponden a la influencia de las fallas temporales sobre el mismo indicador.

El primer término indica que cuando una falla permanente ocurre a lo largo del tramo de red $i$, los usuarios aguas abajo experimentan una interrupción sostenida. El segundo término corresponde a la afectación que produce una falla temporal a lo largo del tramo de red $i$. El tercer término indica que los usuarios aguas arriba de la sección $i$ hasta el reconectador o fusible más cercano, también experimentan una interrupción sostenida. El cuarto término representa el comportamiento de los usuarios aguas arriba del tramo de red $i$ donde se presenta la falla temporal. Es importante aclarar que un fallo temporal a lo largo de la sección $i$ solo se convertirá en fallo permanente si el dispositivo aguas arriba más cercano es un fusible.

\subsection{Función objetivo MAIFI}

El índice MAIFI representa el promedio de las interrupciones temporales del sistema por cliente y se calcula como el cociente entre la suma total de interrupciones temporales percibidas por cada cliente sobre el número total de clientes atendidos. En forma general, la función MAIFI puede calcularse según [18], como se presenta en (3):

$$
M A I F I=\frac{\sum_{I \in L} \gamma_{i} * N_{i}}{N_{t}}=\frac{A_{m}}{N_{t}}
$$

Al igual que se hizo con la ecuación (1), el numerador ubicado en la parte derecha de (3) se puede representar como una ecuación binaria en término de la ubicación de los dispositivos de protección, como se muestra en (4):
De la ecuación (4) se tiene que el primer y segundo término corresponde a las fallas temporales del MAIFI. Una falla permanente a lo largo de la sección se convertirá en falla temporal si el dispositivo de protección aguas arriba más cercano es un fusible con esquema de salvamento. Los usuarios aguas abajo de un fusible sin esquema de salvamento experimentan una falla permanente y esta no contará en el modelo MAIFI. Sin embargo, los clientes protegidos por el reconectador aguas arriba más cercano, experimentan una falla temporal, lo que se ve reflejado en el tercer y cuarto término.

\section{4 Restricciones}

Para el modelo planteado se tienen las siguientes consideraciones previas: los dispositivos están en perfecto estado y están ubicados en las tres fases, las fallas son mutuamente excluyentes e independientes y la topología del sistema es siempre radial e invariante.

Las restricciones del modelo que corresponden a un conjunto de ecuaciones lineales binarias descritas por las ecuaciones (5) a (10), plantean las condiciones descritas a continuación.

La ecuación (5) indica que solo un dispositivo puede ser instalado en un tramo de red, es decir:

$$
x_{i 1}+x_{i 2}+x_{i 4} \geq 2
$$

La ecuación (6) indica si un reconectador o un fusible es requerido en el tramo de red $i$, es decir, tramos de red donde es necesario la presencia de alguno de los dispositivos. En (7) se presenta la restricción que limita el número de elementos en cascada en las trayectorias que van desde la subestación hasta los nodos finales del sistema, con el fin de minimizar la complejidad de coordinación de las protecciones y disminuir el tiempo de operación de los dispositivos:

$$
\begin{gathered}
x_{i 1}+x_{i 2}+x_{i 4}=2 \\
\sum_{t \in I} x_{i 1}+x_{i 2}+x_{i 4} \geq N T_{t}-N C
\end{gathered}
$$

$$
M A I F I=\frac{1}{N_{t}}\left(\begin{array}{c}
\gamma_{1}\left(1-x_{i 1}\right) * N_{i}^{\prime}+\gamma_{i} \sum_{j \in F(i)}\left(1-x_{j 1}\right) * N_{i}^{\prime} \prod_{k \in G(j, i)} x_{k 1} x_{k 2}+ \\
\lambda_{i}\left(1-x_{i 4}\right) \sum_{j \in F(i)}\left(1-x_{j 1}\right)\left(N_{j}^{\prime}-N_{i}^{\prime}\right) \prod_{k \in G^{\prime}(j, i)} x_{k 1} x_{k 2}+ \\
\left(\lambda_{i} \sum_{j \in F(i)}\left(1-x_{j 1}\right) \sum_{k \in G^{\prime}(j, i)}\left(1-x_{k 4}\right)\left(N_{j}^{\prime}-N_{k}^{\prime}\right) \prod_{m \in G(k, j)} x_{m 1} x_{m 2} x_{m 4} \prod_{n \in G^{\prime}(j, i)} x_{n 1} x_{n 2}\right)
\end{array}\right)
$$


La ecuación (8) presenta el caso en que no se pueda lograr la coordinación de diferentes dispositivos, es decir, la coordinación entre un dispositivo $Y$ en la posición $j$ y el dispositivo $Z$ en la posición $k$. En (9) y (10) se presentan las restricciones cuya función es evitar la ubicación de un reconectador aguas abajo de un fusible o para evitar la ubicación de un fusible con esquema de salvamento aguas abajo de un fusible. Es preciso indicar que para las restricciones (9) y (10), la sección $j$ está ubicada aguas debajo de la sección $i$.

$$
\begin{gathered}
x_{j Y}+x_{k z} \geq 1 \\
x_{i 2}+x_{i 4}+x_{j 1} \geq 2 \\
x_{i 2}+x_{j 4} \geq 1
\end{gathered}
$$

Es importante aclarar que el modelo aquí desarrollado es solo aplicable a redes eléctricas radiales, pues carece de sentido hablar de tramos de red aguas abajo o aguas arriba, cuando existe más de un camino hacia la subestación. Adicionalmente, las redes enmalladas, deben ser analizadas con esquemas de protección diferentes a los aquí presentados, puesto que la bidireccionalidad del flujo de potencia implica esquemas de protección bidireccionales, el cual es un abordaje que no está contemplado en este trabajo.

\subsection{Modelo matemático completo}

El modelo matemático completo para la localización óptima de reconectadores y fusibles en sistemas de distribución corresponde a la minimización de los indicadores SAIFI o MAIFI definidos en (2) y (4), sujeto al conjunto restricciones (5) a (10). En forma general ambos modelos matemáticos pueden ser escritos como (11) y (12):

$$
\begin{aligned}
\min S A I F I & \text { Ecuación (2) } \\
\text { s.a. } & \text { Ecuaciones }\{(5) \text { a }(10)\}
\end{aligned}
$$

$$
\begin{aligned}
\min M A I F I & : \text { Ecuación (4) } \\
\text { s.a. } & \text { Ecuaciones }\{(5) \text { a }(10)\}
\end{aligned}
$$

\section{TÉCNICA DE SOLUCIÓN}

El algoritmo genético básico ( $A G)$ fue inicialmente formulado por Holland [20] y está basado en el proceso de selección natural que se presenta en la naturaleza, en el cual los individuos más adaptados tienen más posibilidad de sobrevivir y transmitir su código genético a su descendencia. Matemáticamente un AG es considerado como una técnica de optimización combinatorial, que tiene una alta probabilidad de encontrar las soluciones globales de problemas complejos de gran tamaño y con muchos óptimos locales. Para profundizar en la teoría básica de los AG se pueden consultar [21]-[22].

\subsection{Algoritmo genético de Chu \& Beasley}

En este trabajo el AGCB para resolver el problema de la asignación generalizada de tareas propuesto por Chu \& Beasley [23], es empleado para solucionar el problema de localización óptima de dispositivos de protección en redes radiales de distribución de media tensión, ya que existen gran cantidad de reportes en la literatura especializada de adaptaciones exitosas de esta clase de algoritmos de optimización a sistemas eléctricos.

\subsection{Metodología de construcción de un AGCB}

\subsubsection{Codificación}

Con el fin de representar el problema de localización óptima de reconectadores y fusibles en sistemas de distribución se emplea un esquema de codificación binaria como el presentado en la Fig. 1 , donde el tamaño del vector codificación es tres veces el número de tramos de red del sistema.

Fig. 1. ESQUEMA DE CODIFICACIÓN EMPLEADO

\begin{tabular}{c|c|c|c|c|c|c|c|c|c|c|c|} 
& \multicolumn{1}{c}{ Reconectadores } & \multicolumn{4}{c}{ Fusibles sin esquema de salvamento } & \multicolumn{1}{c}{ Fusibles con esquema de salvamento } \\
1 & 2 & $\cdots$ & $n$ & $n+1$ & $n+2$ & $\ldots$ & $2 n$ & $2 n+1$ & $2 n+2$ & $\ldots$ & $3 n$ \\
\hline 0 & 1 & $\cdots$ & 1 & 0 & 0 & $\cdots$ & 1 & 1 & 0 & $\ldots$ & 0 \\
\hline
\end{tabular}


La primera parte del vector corresponde a la ubicación de los reconectadores, la segunda parte representa los fusibles sin esquema de salvamento, y la última parte corresponde a los fusibles con esquema de salvamento. Cuando en una posición del vector se tiene el valor de cero (0), se indica que el elemento fue instalado en dicha posición, en caso contrario aparecerá un uno (1).

\subsubsection{Población inicial}

Se obtiene generando una parte con algoritmos heurísticos constructivos y la otra parte usando soluciones aleatorias controladas. En lo que corresponde a los algoritmos constructivos, se utiliza un indicador de sensibilidad para obtener una solución aproximada del problema, y a partir de esta solución son introducidas perturbaciones aleatorias en la vecindad, hasta obtener una cantidad predeterminada de soluciones. En cuanto a las soluciones aleatorias controladas se debe tener un conocimiento previo del problema y de las soluciones que pueden aparecer, con el objetivo de guiar el proceso de generación de los valores más adecuados para las variables y de la cantidad de variables de decisión que deben tener valores diferentes de cero. En el caso del AGCB los individuos de la población inicial deben ser diferentes y se debe cumplir con el criterio de diversidad (distancia mínima entre soluciones) [24].

\subsubsection{Criterio de diversidad}

En cualquier ciclo generacional, cada solución de la población debe conservar una distancia mínima de separación respecto a los otros (diversidad controlada), excepto en los casos en que aplica el criterio de aspiración. Con el criterio de diversidad se garantiza una mejor exploración del espacio de soluciones, evitando así la convergencia prematura, siendo esta una gran diferencia respecto al algoritmo genético básico.

\subsubsection{Función objetivo e infactibilidad}

En el AGCB se evalúa para cada individuo una función objetivo y un indicador de infactibilidad. Estas dos se almacenan por separado y se utilizan para propósitos diferentes. La función objetivo se encarga de la selección y de la sustitución de un individuo cuando se tiene una población factible. La infactibilidad se emplea únicamente para susti- tuir un individuo de la población cuando aparecen soluciones infactibles en la población, haciendo esta una de las características más significativas del AGCB porque a diferencia del AG básico, no existe el factor de penalidad por el cual tengan que ser multiplicadas las infactibilidades, por lo que al no involucrar este factor, se evita ingresar al algoritmo valores subjetivos.

\subsubsection{Selección}

El AGCB utiliza el método de selección por torneo, donde se realizan dos torneos para seleccionar los dos padres correspondientes. En cada torneo participan $\mathrm{n}$ individuos (donde $\mathrm{n}$ esta entre 2 y 4). Cada torneo se realiza de la siguiente manera. Se seleccionan $n$ individuos y se comparan sus funciones objetivo y el que tenga la mejor se almacena en la posición que le corresponde, ya sea el padre 1 o el padre 2. Luego de tener los dos padres, ambos pasan al proceso de recombinación.

\subsubsection{Recombinación}

Es el intercambio de material genético de los padres, se hace utilizando cualquier método de recombinación [17-19]. Después de realizada la recombinación se generan dos descendientes, cada uno con material genético de cada uno de los padres, pero solo uno puede avanzar a la siguiente generación y el otro será desechado. La selección del descendiente seleccionado puede ser de forma aleatoria o de forma controlada.

\subsubsection{Mutación}

El descendiente seleccionado corresponde a un vector que contiene algunos valores específicos en cada una de las posiciones asociadas a las variables de decisión del problema. En este proceso de mutación se seleccionan aleatoriamente $n$ de estas posiciones para aumentar o disminuir su contenido. Esta decisión de aumentar o disminuir también puede ser controlada o aleatoria.

\subsubsection{Mejoría local de un individuo}

Puede hacerse de dos formas: mejorando su función objetivo o disminuyendo su infactibilidad. Cualquiera de las dos formas se puede auxiliar de un algoritmo heurístico constructivo basado en la sensibilidad; para el caso de la infactibilidad, 
puede ser usado un algoritmo que identifique elementos atractivos para ser adicionados o retirados para hacer la solución factible.

\subsubsection{Modificación de la población}

En el AGCB solo se cambia un individuo en cada ciclo generacional, siempre y cuando sea de mejor calidad y cumpla el criterio de diversidad. En el AGCB se pueden tener los siguientes escenarios:

- Una población infactible y un descendiente infactible: el descendiente infactible hará parte de la población solo si el nuevo integrante es menos infactible.

- Una población factible y un descendiente infactible: este será eliminado y no hará parte de la siguiente generación.

- Una población infactible y un descendiente factible: este tomará la posición del individuo menos factible de la población.

- Una población factible y un descendiente factible: este tomará la posición del individuo con peor función objetivo solo si este nuevo descendiente posee una mejor función objetivo, de lo contrario, será desechado.

\subsubsection{Criterio de parada}

El AGCB será interrumpido si la incumbente no mejora después de un número especificado de iteraciones o si se satisface el número máximo de ciclos generacionales.

\subsubsection{Definición de parámetros de control}

La efectividad del AGCB para resolver un problema específico depende en buena parte de la codificación, y si ha sido diseñada adecuadamente la población, convergerá hacia una buena solución; sin embargo, esto no garantiza que se encuentre una solución de mejor calidad. Algunas veces, de acuerdo con el tamaño del problema, para encontrar soluciones de mejor calidad se procede a calibrar el comportamiento del AG modificando los parámetros de control (el tamaño de la población, la tasa de crossover $\rho c$ y la tasa de mutación $\rho m$ ).

\subsection{Esquema general de implementación de un AGCB}

En el diagrama de bloques de la Fig. 2 se presenta un esquema general de la implementación y desarrollo de un algoritmo genético para problemas de optimización combinatorial.

Fig. 2. DIAGRAMA DE FLUJO DEL AGCB

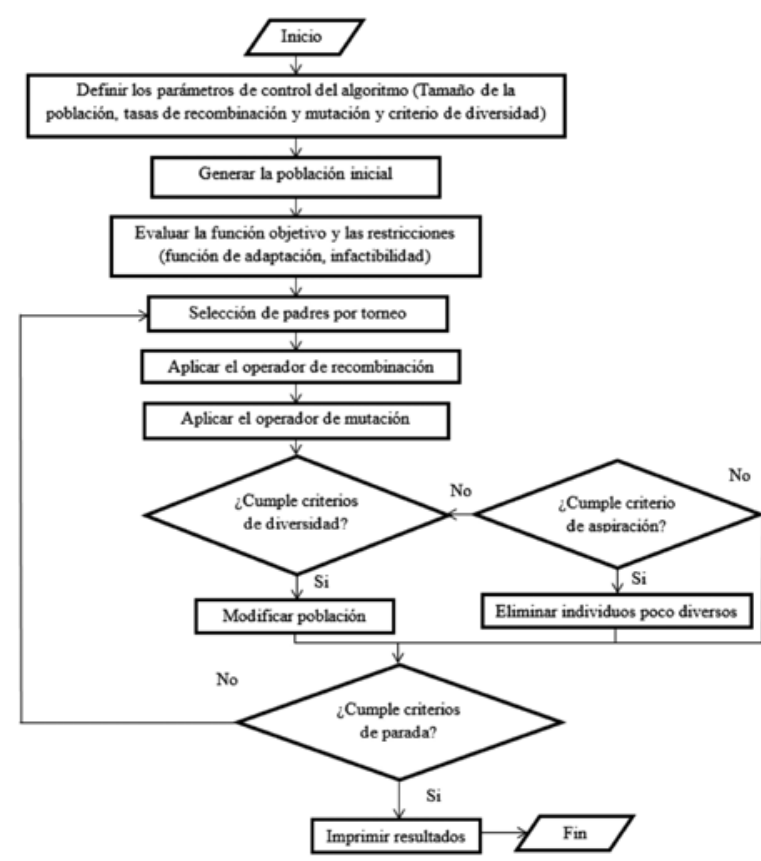

Fuente: autores

\section{METODOLOGÍA PROPUESTA}

A continuación en la Fig. 3 se describe la metodología propuesta para el problema de ubicación óptima de reconectadores y fusibles en sistemas de distribución para el mejoramiento de los índices de confiabilidad SAIFI y MAIFI. Se puede observar que la metodología considera dos estrategias: solucionar el problema empleando el optimizador comercial GAMS o empleando un AGCB. La ventaja de emplear ambas estrategias es que se puede verificar la calidad de las soluciones. Sin embargo, el AGCB permite encontrar soluciones óptimas alternativas diferentes a las encontradas con GAMS.

Es preciso aclarar que los fusibles para ubicar en el sistema de prueba, ya sea para mejorar el índice SAIFI o el índice MAIFI, son de las mismas características, con la particularidad que tiene un esquema de coordinación diferente.

La implementación de la metodología y su aplicación, para los dos sistemas de prueba, se realizó en el software MATLAB y el paquete de optimización comercial GAMS. 
En los dos sistemas se hace un análisis comparativo entre los resultados obtenidos con GAMS y el AGCB con el fin de mostrar la validez y eficiencia de estas estrategias.

Fig. 3. DIAGRAMA DE FLUJO DE LA METODOLOGÍA PROPUESTA

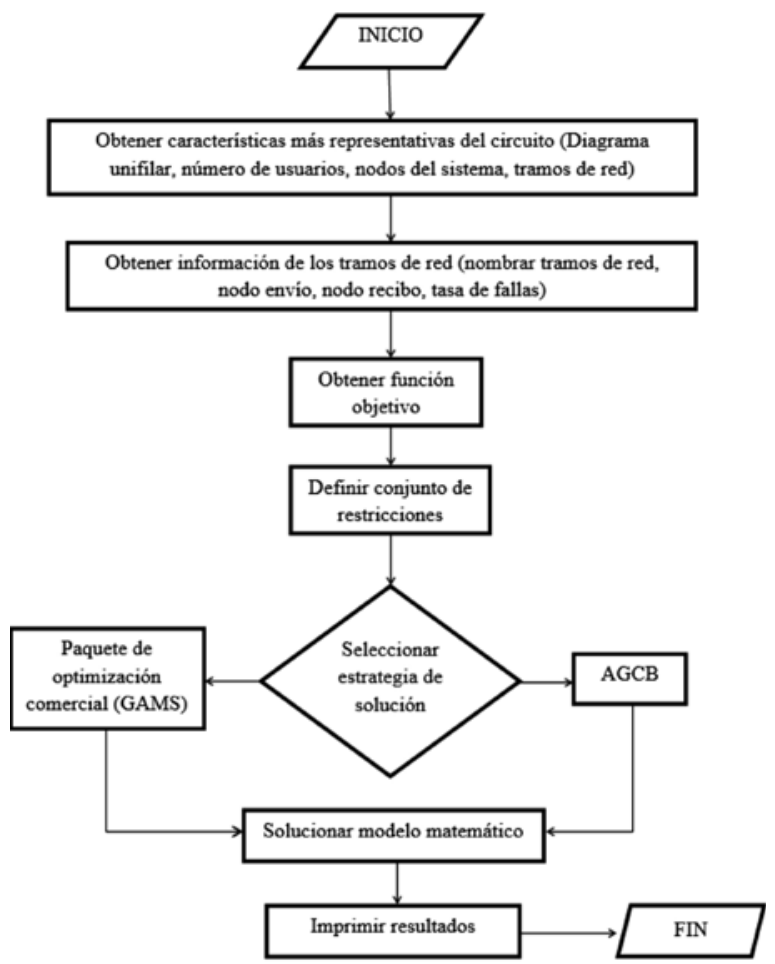

Fuente: autores

\section{APLICACIÓN Y RESULTADOS}

El primer sistema de prueba es comúnmente empleado en la literatura especializada para validar modelos matemáticos, el cual permite un máximo de cuatro reconectadores para instalar y no hay límite en la ubicación de fusibles. Para el segundo sistema permanece libertad de usar un número indefinido de fusibles, pero con la condición de que no pueden instalarse más de seis elementos en cascada para evitar complicaciones en su coordinación, con el fin de reducir los tiempos de operación.

\subsection{Sistema de prueba 1}

Para este sistema, presentado en la Fig. 4 [18], se tiene una red radial compuesta por nueve tramos de red y diez nodos, incluyendo la subes- tación, la cual alimenta un total de 5000 usuarios. Los datos de este se presentan en la Tabla I.

Fig. 4. SISTEMA DE PRUEBA DE 9 SECCIONES

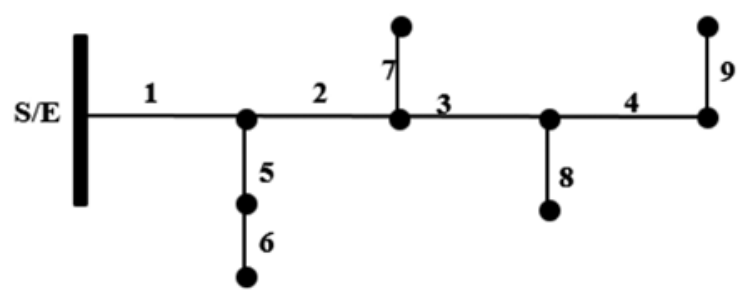

Fuente: autores

En la implementación del AGCB se emplea una población de 1000 individuos, un número de generaciones variable entre 50000 y 100000 , con una tasa de mutación de 0.05 y una tasa de recombinación de 0.95 .

\subsubsection{Indicador SAIFI}

Al aplicar el modelo de optimización descrito en (12) se obtiene las respuestas presentadas en la Tabla II a través de la valoración del modelo en GAMS y con el AGCB.

En la Tabla II se observan los resultados obtenidos con las dos técnicas de solución. Se observa que varía la ubicación de los dispositivos de protección, pero se mantiene el mismo valor de función objetivo, es decir, presenta óptimos alternativos.

Con el AGCB, además de encontrar la solución dada por GAMS, también se encuentran otras soluciones en las que la ubicación de dispositivos es diferente, pero con igual valor de función objetivo (ver Tabla III). De acuerdo con esto, depende del operador de red seleccionar cuál se ajusta más a sus necesidades, en función de otras variables que no están contenidas en este trabajo.

De acuerdo con los resultados obtenidos en este sistema de prueba, se observa que para mejorar el índice SAIFI es necesario implementar fusibles para salvamento en vez del otro tipo de fusibles, pues los fusibles para salvamento hacen ver la interrupción de una forma temporal, es decir, disminuye el número de fallas permanentes.

\subsubsection{Indicador MAIFI}

Para este índice se obtienen también varias respuestas, entre ellas la respuesta dada por el 
paquete de optimización comercial. En la Tabla IV se muestran los resultados obtenidos con las dos estrategias de solución. Se observa que varía la ubicación de dispositivos, aunque con igual valor de la función objetivo.

De acuerdo con los resultados obtenidos, se observa que para mejorar el índice MAIFI es necesario implementar un esquema en el cual no se tienen en cuenta los fusibles para salvamento, ya que estos hacen que las interrupciones que ocurren en el sistema sean temporales.
Los fusibles por ubicar en el sistema de prueba, ya sea para mejorar el índice SAIFI o el índice MAIFI, son de las mismas características, con la particularidad de que tienen un esquema de coordinación diferente.

Después de analizar este sistema de prueba se observa que para los índices SAIFI o MAIFI es más eficiente el AGCB, ya que proporciona más soluciones que un paquete de optimización comercial, dando así más opciones a las empresas prestadoras del servicio eléctrico.

TABLA I

DATOS DEL SISTEMA DE PRUEBA 1

\begin{tabular}{|c|c|c|c|c|c|}
\hline Sección & $\begin{array}{c}\text { Nodo de } \\
\text { envío }\end{array}$ & $\begin{array}{c}\text { Nodo de } \\
\text { recibo }\end{array}$ & $\begin{array}{c}\text { Tasa de fallas permanentes } \\
\text { [fallas/año/km] }\end{array}$ & $\begin{array}{c}\text { Tasa de fallas temporales } \\
\text { [fallas/año/km] }\end{array}$ & $\begin{array}{c}\text { Número de consumidores } \\
\text { por tramo }\end{array}$ \\
\hline 1 & 1 & 2 & 0,8 & 1,2 & 800 \\
\hline 2 & 2 & 3 & 0,8 & 1,4 & 1200 \\
\hline 3 & 3 & 4 & 0,9 & 1,6 & 800 \\
\hline 4 & 4 & 9 & 0,7 & 1 & 600 \\
\hline 5 & 2 & 5 & 0,9 & 2 & 500 \\
\hline 6 & 5 & 6 & 0,7 & 1,7 & 300 \\
\hline 7 & 3 & 7 & 0,8 & 2,8 & 400 \\
\hline 8 & 4 & 8 & 1 & 3,2 & 200 \\
\hline 9 & 9 & 10 & 0,5 & 0,7 & 200 \\
\hline
\end{tabular}

Fuente: autores

TABLA II

RESPUESTA PARA EL ÍNDICE SAIFI EN EL SISTEMA DE PRUEBA 1

\begin{tabular}{|c|c|c|c|c|}
\hline \multirow{2}{*}{ Estrategia de solución } & \multicolumn{3}{|c|}{ Ubicación de dispositivos } & \multirow{2}{*}{ SAIFI } \\
\cline { 2 - 5 } & Reconectador & Fusible & Fusible para salvamento & \\
\hline AGCB & $1,3,4,9$ & -- & $5,6,7,8$ & 2.346 \\
\hline GAMS & $1,3,4,5$ & - & $6,7,8,9$, & 2.346 \\
\hline
\end{tabular}

Fuente: autores

TABLA III

RESPUESTAS ADICIONALES PARA EL ÍNDICE SAIFI EN EL SISTEMA DE PRUEBA 1

\begin{tabular}{|c|c|c|c|c|}
\hline \multirow{2}{*}{$\begin{array}{c}\text { Óptimos alternativos en- } \\
\text { contrados con el AGCB }\end{array}$} & \multicolumn{3}{|c|}{ Ubicación de dispositivos } & \multirow{2}{*}{ SAIFI } \\
\cline { 2 - 5 } & Reconectador & Fusible & Fusible para salvamento & 2.346 \\
\hline Respuesta adicional 1 & $1,3,4$ & - & $5,6,7,8,9$ & 2.346 \\
\hline Respuesta adicional 2 & $1,3,4,7$ & - & $5,6,8,9$ & 2.346 \\
\hline Respuesta adicional 3 & $1,3,4,8$ & - & $5,6,7,9$ & 2 \\
\hline
\end{tabular}

Fuente: autores

TABLA IV

RESPUESTA PARA EL ÍNDICE MAIFI EN EL SISTEMA DE PRUEBA 1

\begin{tabular}{|c|c|c|c|c|}
\hline \multirow{2}{*}{ Estrategia de solución } & \multicolumn{3}{|c|}{ Ubicación de dispositivos } & \multirow{2}{*}{ MAIFI } \\
\cline { 2 - 5 } & Reconectador & Fusible & Fusible para salvamento & \\
\hline AGCB & $1,3,4$ & $5,7,8,9$ & - & 3.336 \\
\hline GAMS & $1,3,4$ & $5,6,7,8,9$ & - & 3.336 \\
\hline
\end{tabular}

Fuente: autores 


\subsection{Sistema de prueba 2}

Este sistema pertenece a la compañía de distribución de Qazvin en Irán [19], el cual está compuesto por cuarenta y nueve tramos de red y cincuenta nodos que alimentan un total de 3413 usuarios. Su topología y características pueden ser consultadas en [19].

Para la implementación del AGCB se emplea una población de 1000 individuos y un rango de generaciones entre 50000 y 100000 . Las tasas de mutación y de recombinación son 0.05 y 0.95 , respectivamente.

Al igual que con el sistema de prueba 1 , se desarrolla la metodología propuesta en el capítulo 4 para calcular las tasas de falla permanentes y temporales, la cantidad de usuarios atendidos por cada tramo de red, la carga por cada tramo de red y el diagrama del sistema. Se incrementa el vector que contiene a los tramos de red en los cuales no pueden ser instalados fusibles, ya sea porque pertenecen a la sección principal o porque son nodos en los cuales la cantidad de usuarios conectados es nula.

\subsubsection{Indicador SAIFI}

En la Tabla $V$ se observan los resultados obtenidos con las dos técnicas de solución, en las cuales varía la ubicación de dispositivos, pero se conserva el mismo valor de función objetivo.

Se observa en los resultados de la Tabla VI que el AGCB propone una solución más eficiente que la propuesta por el paquete de optimización comercial (GAMS), pues aparte de encontrar una solución óptima con el mismo valor de función objetivo, la solución que encuentra propone la ubicación de un fusible menos, por lo cual, desde el punto de vista económico, es mejor la solución dada por la metaheurística.

\subsubsection{Indicador MAIFI}

A continuación se muestran las respuestas obtenidas por las dos técnicas de solución empleadas, para la minimización del índice MAIFI. Es importante repetir que para este sistema se tiene una consideración adicional, la cual limita el número de elementos en cascada a seis.

Los resultados obtenidos en el sistema de prueba 2 tienen un comportamiento similar al sistema de prueba 1, en el cual para la minimización de este índice se ubican fusibles cuyo esquema de coordinación no emplea salvamento.

Con el AGCB, además de encontrar la solución dada por GAMS, también encuentra otras soluciones en las que la ubicación de dispositivos es diferente para todas las propuestas, pero el valor de función objetivo es el mismo (ver Tabla VII). De acuerdo con esto, depende de la empresa distribuidora seleccionar cuál se ajusta más a sus necesidades, en función de otras variables que no están contenidas en este proyecto.

Además, se tienen ubicaciones en las que el número de dispositivos instalados es menor que en la respuesta dada por el paquete de optimización comercial, siendo esto, un beneficio económico para la empresa prestadora del servicio.

TABLA V

RESPUESTA PARA EL ÍNDICE SAIFI EN EL SISTEMA DE PRUEBA 2

\begin{tabular}{|c|c|c|c|c|}
\hline \multirow{2}{*}{ Estrategia de solución } & \multicolumn{3}{|c|}{ Ubicación de dispositivos } & \multirow{2}{*}{ SAIFI } \\
\cline { 2 - 6 } & Reconectador & Fusible & Fusible salvamento & 6.3030 \\
\hline AGCB & $1,8,14,33$ & - & $19,20,23,24,26,29,31,32,34,35,38,39,40,41,42,43,44,45,46$ & 6 \\
\hline GAMS & $1,8,14,33$ & - & $19,20,23,24,26,29,31,32,34,35,37,38,39,40,41,42,43,44,45,46$ & 6.3030 \\
\hline
\end{tabular}

Fuente: autores

TABLA VI

RESPUESTA PARA EL ÍNDICE MAIFI EN EL SISTEMA DE PRUEBA 2

\begin{tabular}{|c|c|c|c|c|}
\hline \multirow{2}{*}{ Estrategia de solución } & \multicolumn{3}{|c|}{ Ubicación de dispositivos } & \multirow{2}{*}{ MAIFI } \\
\cline { 2 - 5 } & Reconectador & Fusible & Fusible para salvamento & \\
\hline AGCB & $1,8,14,18$ & $19,24,26,29,32,34,35,39,41,44,45,46$ & - & 7.8374 \\
\hline GAMS & $1,8,14,18$ & $19,24,26,29,32,34,35,39,41,44,45,46$ & - & 7.8374 \\
\hline
\end{tabular}

Fuente: autores 
TABLA VII

RESPUESTAS ADICIONALES PARA EL ÍNDICE SAIFI EN EL SISTEMA DE PRUEBA 2

\begin{tabular}{|c|c|c|c|c|}
\hline \multirow{2}{*}{$\begin{array}{c}\text { Óptimos alternativos } \\
\text { encontrados con el } \\
\text { AGCB }\end{array}$} & \multicolumn{3}{|c|}{ Ubicación de dispositivos } & \multirow{2}{*}{ MAIFI } \\
\hline & Reconectador & Fusible & Fusible para salvamento & \\
\hline Respuesta adicional 1 & $1,8,14,18$ & $19,23,24,26,29,32,34,35,39,41,44,45,46$ & -- & 7.8374 \\
\hline Respuesta adicional 2 & $1,8,14,18$ & $\begin{array}{c}19,23,24,26,29,31,32,34,35,3839,41,42,43,44 \\
45,46\end{array}$ & -- & 7.8374 \\
\hline Respuesta adicional 3 & $1,8,14,18$ & $19,23,24,26,29,34,41,45,46$ & - & 7.8374 \\
\hline Respuesta adicional 4 & $1,8,14,18$ & $19,24,26,29,34,35,37,38,40,41,42,44,45,46$ & - & 7.8374 \\
\hline Respuesta adicional 5 & $1,8,14,18$ & $19,23,24,29,31,32,34,35,39,41,43,44,45,46$ & - & 7.8374 \\
\hline Respuesta adicional 6 & $1,8,14,18$ & $19,24,29,34,38,41,42,44,46$ & - & 7.8374 \\
\hline
\end{tabular}

Fuente: autores

\section{CONCLUSIONES}

Para solucionar el problema de ubicación óptima de reconectadores y fusibles en sistemas de distribución que minimicen los índices de confiabilidad SAIFI y MAIFI, se propone una metodología que emplea como técnica de solución el AGCB, el cual, a partir de una población de individuos factible, evoluciona para encontrar la respuesta óptima; es decir, una ubicación recomendable de dispositivos de protección en el sistema bajo estudio. Además se emplea el paquete de optimización comercial GAMS, con el fin de comparar los resultados obtenidos y así, demostrar la eficiencia del AGCB.

El uso de técnicas metaheurísticas para la solución de problemas de gran tamaño y un nivel de complejidad alto como es el caso de los sistemas eléctricos, permite obtener un conjunto de soluciones alternativas adicionales a la solución óptima obtenida por un paquete de optimización comercial, permitiéndole al usuario, en este caso el operador de red, inclinarse por la respuesta que más se acomode a sus necesidades.

La metodología propuesta es flexible, ya que puede ser implementada en diferentes sistemas de distribución independientemente de su tamaño, siempre y cuando se tenga una topología radial.

Se demuestra la importancia que tiene una buena coordinación de dispositivos de protección y se verifica la utilidad de implementar fusibles con esquema de salvamento, con el fin de reducir índices de confiabilidad como es el caso del SAIFI, para evitar sanciones por parte de las entidades.
Se modificó el modelo matemático del problema presentado en [18], y se agregó un conjunto de restricciones asociadas al máximo número de elementos en cascada que pueden ser ubicados sobre el alimentador y sus laterales para evitar problemas de coordinación entre dispositivos y tener un mayor control en los tiempos de operación de estos.

\section{REFERENCIAS}

[1] M. T. G. Donalvo, A. E. D. Tio and W. R. D. Tarnate, "Maximizing reliability by optimal siting of distributed generation and protective devices," Environment and Electrical Engineering (EEEIC), 2015 IEEE 15th International Conference on, Rome, 2015, pp. 455-460. Doi: 10.1109/EEEIC.2015.7165205.

[2] B. Baruah, S. Das and M. Ghosh, "Optimal placement of protective devices in a distributed power system," 2015 Annual IEEE India Conference (INDICON), New Delhi, 2015, pp. 1-6. doi: 10.1109/INDICON.2015.7443358.

[3] Yuting Tian and J. Mitra, "A pareto-optimal approach to design of distribution system protection," North American Power Symposium (NAPS), 2015, Charlotte, NC, 2015, pp. 1-5. doi: 10.1109/NAPS.2015.7335250.

[4] Kagan N., Prieto H., Barioni C., Robba J., "Otimização da Qualidade de Fornecimento pela Localização de Dispositivos de Proteção e Seccionamento em Redes de Distribuição", Anais do II Seminário Brasileiro Sobre Qualidade de Energia Elétrica (Digital -CD), 16 a 19 de Novembro de 1997, São Lourenço - MG.

[5] F. Soudi and K. Tomsovic, "Optimized distribution protection using binary programming," in IEEE Transactions on Power Delivery, vol. 13, no. 1, pp. 218-224, Jan 1998. doi: 10.1109/61.660881. 
[6] F. Soudi and K. Tomsovic, "Optimal trade-offs in distribution protection design," in IEEE Transactions on Power Delivery, vol. 16, no. 2, pp. 292-296, Apr 2001. doi: $10.1109 / 61.915498$.

[7] N. Kagan and C. C. B. Oliveira, "Goal planning and risk analysis for distribution reliability indices," Electricity Distribution, 2001. Part 1: Contributions. CIRED. 16th International Conference and Exhibition on (IEE Conf. Publ No. 482), Amsterdam, 2001, pp. 6 pp. vol.2-. doi: 10.1049/cp:20010752.

[8] Silva, L.G.W. Alocação otimizada de dispositivos de proteção em sistemas de distribuição de energia elétrica. Dissertação (Mestrado) - Faculdade de Engenharia de Ilha Solteira, Universidade Estadual Paulista, Ilha Solteira, 2002.

[9] Silva, L.G.W. Desenvolvimento de uma metodologia integrada para alocação otimizada de dispositivos de controle e proteção previsão de carga em sistemas de energia elétrica. Dissertação (Doutorado) - Faculdade de Engenharia de Ilha Solteira, Universidade Estadual Paulista, Ilha Solteira, 2005.

[10] Moreti, E. Análises e estudos para alocação e ajustes de dispositivos de proteção em redes de média tensão de energia elétrica com geração Distribuída. Dissertação (Mestrado) - Faculdade de Engenharia de Ilha Solteira, Universidade Estadual Paulista, Ilha Solteira, 2007.

[11] Reyes J., Morales M., Garcia-Santander L., Pezoa J. E., "Optimal location of reclosers in distribution networks in order to minimize the average interruption frequency". Revista: Ingeniare, Vol 17, No 2, April 2009 pp. 205-212.

[12] M. H. Kim, S. H. Lim, J. F. Moon and J. C. Kim, "Method of Recloser-Fuse Coordination in a Power Distribution System With Superconducting Fault Current Limiter," in IEEE Transactions on Applied Superconductivity, vol. 20, no. 3, pp. 1164-1167, June 2010. doi: 10.1109/ TASC.2010.2040821.

[13] G. D. Ferreira, A. S. Bretas and G. Cardoso, "Optimal distribution protection design considering momentary and sustained reliability indices," Modern Electric Power Systems (MEPS), 2010 Proceedings of the International Symposium, Wroclaw, 2010, pp. 1-8.

[14] F. Darabi, A. T. Shooshtari, E. Babaei and S. Darabi, "Reliability cost allocation of protective devices using binary programming," Business, Engineering and Industrial Applications (ISBEIA), 2011 IEEE Symposium on, Langkawi, 2011, pp. 469-474. doi: 10.1109/ISBEIA.2011.6088860.
[15] C. Toro, R. A. Hincapié, R. A. Gallego, “Ubicación Óptima de Elementos de Protección en Sistemas de Distribución de Energía Eléctrica," Revista Scientia Et Technica, vol. XVI, núm. 48, agosto, 2011, pp. 13-18. Universidad Tecnológica de Pereira. Pereira, Colombia.

[16] G. D. Ferreira, A. S. Bretas, "A Nonlinear Programming Model for Electric Distribution System Reliability Optimization". Department of Electrical Engineering, Federal University of Rio Grande do Sul, Mayo 2012.

[17] C. A. Peñuela, "Desemvolvimento de Técnicas e Modelos Matemáticos para Solução do Problema do Manejamento da Expansão e Operação de Sistemas de Distribuçã de Energia Eléctrica Com Geradores Distribuídos". Universidade Estadual Julio de Mesquita Filho, Facultade de Engenharia, Campus Ilha Solteira, 2012.

[18] A. E. D. C. Tio, I. B. N. C. Cruz, B. M. Malquisto and R. D. del Mundo, "A binary programming model for reliability optimization considering fuse-blow and fusesave schemes," TENCON 2012 - 2012 IEEE Region 10 Conference, Cebu, 2012, pp. 1-6. doi: 10.1109/TENCON.2012.6412247.

[19] Sh. Abdi, K. Afshar, S. Ahmadi, N. Bigdeli, M. Abdi, "Optimal recloser and sectionalizer allocation in distribution networksusing IPSO-Monte Carlo approach". Imam Khomeini International University, Electrical Engineering Department, 2013.

[20] J. H. Holland, "Adaptation in natural and artificial system: An introductory analysis with applications to biology, control and artificial intelligence". The University of Michigan Press, 1975.

[21] D. E. Goldberg, "Genetics Algorithms in search optimization and machine Learning," Addison Wesley Reading, Mass, 1989.

[22] Z. Michalewicz, "Genetics Algorithms + data structure evolution programs," Artificial Intelligence, Springer, Berlin, 1996.

[23] P.C. Chu and J.E. Beasly, "A Genetic Algorithm for the generalized assignment problem," Computers Operation Research, 24(1), pp 17-23, 1997.

[24] R. A. Gallego; A. H. Escobar., E. Toro. "Técnicas metaheurísticas de optimización," 2 ed. Pereira: Universidad Tecnológica de Pereira, 2008. 